\title{
Estilos y estrategias de afrontamiento y rendimiento académico: una revisión empírica
}

\author{
Alix Sofía Ávila Quiñones ${ }^{1}$, Gloria Johanna Montaña², David Jiménez Arenas ${ }^{3}$ \& \\ Jenny Paola Burgos ${ }^{4}$
}

\begin{abstract}
This is a descriptive and detailed revision of qualitative investigations of coping styles and strategies; academic performance of adolescent students. It considered both, independent and correlational studies among the two variables. Different data bases were revised like Dialnet, Redalyc, Scieloy Science Direct. 479 articles were obtained from these, 46 of these responded to the selection criteria that entailed (research article, indexed journal, adolescents). Alongside this search there was a prevalence of the studies related to different variables and their relationship with coping styles and strategies. The studies present common results in terms of positive correlations between active coping styles and well being.
\end{abstract}

\section{Editado por}

William Alejandro Jiménez-Jiménez

Universidad de Boyacá, Colombia.

\section{Revisado por}

Andrés Manuel Pérez-Acosta, Universidad del Rosario, Colombia.

Keywords I Coping styles coping strategies, academic performance, undergraduate students.

\section{${ }^{1}$ Alix Sofía Ávila Quiñones $[\bowtie]$}

${ }^{2}$ Gloria Johanna Montaña

${ }^{3}$ David Jiménez Arenas

4 Jenny Paola Burgos
Psicóloga. Especialista en Psicología del Consumidor. Docente Universidad de Boyacá. email: asavila@uniboyaca.edu.co

Psicóloga. Especialista en Evaluación y Diagnóstico Neuropsicológico. Docente Universidad de Boyacá. email: gjmontana@uniboyaca.edu.co

Psicólogo. Candidato a Maestría en psicoanálisis subjetividad y cultura. Docente Universidad de Boyacá. email: drjimenez@uniboyaca.edu.co

Psicóloga. Maestría en Psicología Clínica y de la Familia. Docente Universidad de Boyacá. email: jepaoburgos@uniboyaca.edu.co 


\section{Introducción}

El objetivo de esta revisión es reconocer la relación de la variable estilos y estrategias de afrontamiento en contextos de educación superior. Los estilos de afrontamiento se definen como "aquellos esfuerzos cognitivos y conductuales constantemente cambiantes que se desarrollan para manejar las demandas específicas externas y/o internas que son evaluadas como excedentes o desbordantes de los recursos del individuo" (Lazarus y Folkman, 1986, p. 164). La variable "estilos" ha sido ampliamente estudiada y puesta en relación con diferentes categorías de análisis como: adaptación, estrés, variables sociodemográficas, bienestar psicológico, personalidad, inteligencia, depresión y rendimiento académico.

Como en la investigación de Morales y Trianes (2010) señalan que el empleo de estrategias de afrontamiento de aproximación a los problemas se asocia a un mayor grado de ajuste y adaptación, mientras que un mayor empleo de estrategias de tipo evitativo se relaciona con menos ajuste y comportamientos más desadaptativos. De igual forma Fernández y Manga (2009) consideran que la madurez, las estrategias de afrontamiento adaptativas y la alta autoestima logran debilitar agentes estresores favoreciendo un afrontamiento activo, utilizando estrategias como aceptación, planificación, reinterpretación positiva y humor.

Cornejo y Lucero (2005) por su parte encontraron que las estrategias de afrontamiento como la solución de problemas y la búsqueda de apoyo social se asocian con bienestar psicológico; Figueroa Contini, Lacunza, Levín y Suedan (2005) también dan cuenta de una correlación positiva entre bienestar psicológico y el uso de estrategias de afrontamiento que implican un papel activo por parte del sujeto. Los adolescentes con bajo bienestar tienden a utilizar estrategias de afrontamiento del tipo pasivo ya que no requieren de empeño por parte del sujeto, sino una evitación del problema y el alivio de la tensión.

Estas tendencias investigativas dejan en evidencia una hipótesis sobre la relevancia que se le otorga a los estilos y estrategias de afrontamiento en las diferentes áreas del comportamiento humano, supuesto que desde lo empírico se sustenta y deja un promisorio trabajo por realizar, dado que consolida recursos efectivos en la intervención psicológica. 
La literatura reporta la relación existente entre los estilos y estrategias de afrontamiento con diversidad de temas y es alrededor de esas posibilidades que se eligió dar relevancia al rendimiento académico. Este estudio inicia con la comprensión de que la transición de la educación básica secundaria a la educación superior trae consigo cambios en los estilos de vida, que plantean retos para la adaptación de los jóvenes en procesos emocionales, familiares, sociales y académicos, siendo necesario estudiar las diferencias individuales de los jóvenes quienes implementan recursos cognitivos y comportamentales para favorecer el logro de sus metas, mientras otros por el contrario presentan dificultades en el rendimiento académico que interfiere con la planeación y ejecución de su proyecto de vida.

Sobre este tema Yengle (2009) encontró que la deserción estudiantil se produce en los primeros tres o cuatro semestres o ciclos de estudio, principalmente en el primer semestre. La dificultad de adaptación está relacionada con la interacción de los estudiantes con sus compañeros de estudio. Por su parte trabajos como los de Aldana, Pérez y Rodríguez (2010) y Tejedor, García y Muñoz (2007) amplían su comprensión sobre el fenómeno del bajo rendimiento académico como multicausal teniendo presente la responsabilidad de los diferentes actores: estudiantes, institución y directivos y docentes y plantean estrategias para superarlo destacándose la motivación al estudiantado, la orientación profesional y la capacitación permanente por parte de los docentes.

También se analizaron investigaciones que tenían por objeto relacionar las variables estilos y estrategias de afrontamiento y rendimiento académico, como el de Frydenberg, Lewis, Kennedy, Ardila, Frindte y Hannoun (2003) donde se encontró que el uso de estrategias como la solución de problemas, esfuerzo y dedicación favorecía el rendimiento académico; mientras que comportamientos de tipo evitativo y síntomas de estrés y ansiedad no lo hacían. Así también lo soporta Martínez (2010) en su investigación donde encontró que los alumnos que se esfuerzan y se comprometen para afrontar el estrés académico tienden a obtener mejor rendimiento escolar. Por el contrario, los estudiantes universitarios que no afrontan los problemas o que los ignoran, tienden a obtener deficientes resultados académicos.

Finalmente esta revisión empírica logró relacionar diversos trabajos investigativos alrededor de los Estilos y estrategias de afrontamiento y diferentes variables asociadas y conectarlo con el rendimiento académico de estudiantes universitarios animando una mirada multi-causal que favorece su comprensión e intervención interdisciplinar en escenarios educativos. 


\section{Metodología}

Esta revisión comprende un estudio descriptivo de análisis de documentos de corte empírico, desde un enfoque interpretativo. El rango de búsqueda de los artículos se estableció entre los años 2001 - 2013, indagando las bases de datos Dialnet, Scielo, Psique y Redalyc. Se obtuvo un total de 479 artículos, de los cuales 46 cumplieron los siguientes criterios de inclusión: artículos empíricos publicados en revistas indexadas, artículos publicados en el idioma español, y población adolescente escolarizada. Las palabras clave de búsqueda fueron: estilos y estrategias de afrontamiento, rendimiento académico y estudiantes universitarios.

Las categorías de análisis de los artículos fueron tres:

1. Estilos y estrategias de afrontamiento y variables asociadas. Esta categoría refiere la descripción y el análisis de los artículos empíricos que relacionan los estilos y estrategias de afrontamiento con temas como adaptación, auto-concepto y auto-estima, personalidad, ansiedad y depresión, bienestar psicológico, género, capacidad intelectual y cultura.

2. Rendimiento académico. Esta categoría describe y analiza los artículos que referencian investigaciones relacionadas con factores de tipo social, personal, familiar, entre otros; que inciden en el bajo rendimiento académico.

3. Estilos y estrategias de afrontamiento y rendimiento académico. En esta unidad de análisis se incluyen los artículos empíricos que vinculan la relación entre las variables citadas.

\section{Resultados}

Los resultados obtenidos se presentan de acuerdo a las unidades o categoría establecidas. Cada categoría constará de la síntesis y la descripción de los artículos encontrados y su posterior comprensión y análisis.

\subsection{Estilos y Estrategias de Afrontamiento}

En esta categoría se encontraron 24 artículos de diseño metodológico correlacional. En general, estos documentos señalan una relación positiva entre estrategias de afrontamiento 
de tipo activo con variables como bienestar psicológico, adaptación, inteligencia, auto concepto y autoestima. En la tabla 1 se presentan los artículos que relacionan los estilos y estrategias de afrontamiento con otras variables.

\begin{tabular}{|c|c|c|c|}
\hline Categoría & Autores & Instrumentos & Resultados \\
\hline \multirow[t]{4}{*}{ Adaptación } & $\begin{array}{l}\text { Arjona y Guerre- } \\
\text { ro (2001) }\end{array}$ & $\begin{array}{l}\text { "Escala de Afrontamiento } \\
\text { (ACS)", "Inventario de Adap- } \\
\text { tación de Conducta" (IAC) y } \\
\text { la "Escala Multidimensional } \\
\text { de Expresión Social-Parte } \\
\text { Motora". }\end{array}$ & $\begin{array}{l}\text { Las estrategias de afrontamiento denominadas acti- } \\
\text { vas como la búsqueda de soluciones correlacionan } \\
\text { con las habilidades sociales y con la adaptación en } \\
\text { los sujetos. Las estrategias de afrontamiento pasi- } \\
\text { vas (evitación), se relacionan negativamente, con la } \\
\text { adaptación global del sujeto. }\end{array}$ \\
\hline & $\begin{array}{l}\text { Moradi y Cols } \\
\text { (2011) }\end{array}$ & $\begin{array}{l}\text { CRI (Inventario de respuestas } \\
\text { de afrontamiento) }\end{array}$ & $\begin{array}{l}\text { La inteligencia emocional tiene relación con estra- } \\
\text { tegias de afrontamiento tanto efectivas como no } \\
\text { efectivas, no es la única variable asociada a la elec- } \\
\text { ción de unas u otras estrategias. }\end{array}$ \\
\hline & $\begin{array}{l}\text { Morales y Tria- } \\
\text { nes (2010) }\end{array}$ & $\begin{array}{l}\text { ESAN (Estrategias situaciona- } \\
\text { les de afrontamiento en niños } \\
\text { y en niñas) }\end{array}$ & $\begin{array}{l}\text { Se concluye que el empleo de estrategias de } \\
\text { afrontamiento de aproximación a los problemas se } \\
\text { asocia a un mayor grado de ajuste y adaptación, } \\
\text { mientras que un mayor empleo de estrategias de } \\
\text { tipo evitativo se relaciona con menos ajuste y com- } \\
\text { portamientos más desadaptativos. }\end{array}$ \\
\hline & $\begin{array}{l}\text { Fraguela, Luen- } \\
\text { go, Romero, } \\
\text { Villar y Sobral } \\
\text { (2006) }\end{array}$ & $\begin{array}{l}\text { Escala de afrontamiento para } \\
\text { adolescentes }\end{array}$ & $\begin{array}{l}\text { Los resultados indican que algunas estrategias } \\
\text { actúan como factores de protección para la salud, } \\
\text { mientras que otras favorecen la aparición de con- } \\
\text { ductas antisociales. }\end{array}$ \\
\hline $\begin{array}{l}\text { Autoconcepto } \\
\text { y autoestima }\end{array}$ & $\begin{array}{l}\text { Barrón, Castilla, } \\
\text { Casullo y Verdú } \\
\text { (2002) }\end{array}$ & $\begin{array}{l}\text { "Escala de Afrontamiento } \\
\text { (ACS)" y escala BIEPS. }\end{array}$ & $\begin{array}{l}\text { Los autores confirmaron la relación positiva entre } \\
\text { autoconcepto positivo, estilo de afrontamiento } \\
\text { activo y satisfacción con la vida. }\end{array}$ \\
\hline
\end{tabular}




\begin{tabular}{|c|c|c|c|}
\hline & $\begin{array}{l}\text { Fernández y } \\
\text { Manga (2009) }\end{array}$ & $\begin{array}{l}\text { El Cuestionario de Sentido de } \\
\text { Coherencia (SOC-13), Cues- } \\
\text { tionario de Estrés Percibido, } \\
\text { La Escala de Cansancio Emo- } \\
\text { cional (ECE), El Cuestionario } \\
\text { de Afrontamiento (COPE-28), } \\
\text { El Cuestionario de Salud } \\
\text { General y El Cuestionario } \\
\text { Revisado de Personalidad de } \\
\text { Eysenck (EPQ-R). }\end{array}$ & $\begin{array}{l}\text { Se encuentra relación entre madurez y las estrate- } \\
\text { gias de afrontamiento adaptativas que debilitan el } \\
\text { estresor. La relación es negativa con estrategias no } \\
\text { adaptativas. Por otra parte, las personas con alta } \\
\text { autoestima presentan Afrontamiento activo, utili- } \\
\text { zando estrategias como Aceptación, Planificación, } \\
\text { Reinterpretación positiva y Humor. Se encontró una } \\
\text { relación negativa entre autoestima y estrategias de } \\
\text { afrontamiento como Negación, Autoinculpación y } \\
\text { Desconexión conductual. }\end{array}$ \\
\hline \multirow[t]{3}{*}{ Personalidad } & $\begin{array}{l}\text { Cassaretto } \\
(2010)\end{array}$ & $\begin{array}{l}\text { Inventario de Personalidad } \\
\text { NEO y, el Inventario de Esti- } \\
\text { mación del Afrontamiento: } \\
\text { COPE. }\end{array}$ & $\begin{array}{l}\text { Los resultados arrojaron asociaciones importantes } \\
\text { y significativas entre los estilos de afrontamiento } \\
\text { y los rasgos de personalidad, por ejemplo, a ma- } \\
\text { yor neuroticismo menor esfuerzo para resolver el } \\
\text { estresor, desarrollando una actitud pasiva ante los } \\
\text { problemas. }\end{array}$ \\
\hline & $\begin{array}{l}\text { Castaño y León } \\
\text { del Barco (2010) }\end{array}$ & $\begin{array}{l}\text { Escalas de Adjetivos Inter- } \\
\text { personales y Estrategias de } \\
\text { Afrontamiento (Coping Stra- } \\
\text { tegies Inventory, CSI. }\end{array}$ & $\begin{array}{l}\text { Los sujetos modifican sus estrategias en función de } \\
\text { la situación, pero no en lo que respecta a las carac- } \\
\text { terísticas de personalidad. }\end{array}$ \\
\hline & $\begin{array}{l}\text { Contreras y Espi- } \\
\text { noza (2009) }\end{array}$ & $\begin{array}{l}\text { Inventario de personalidad } \\
\text { NEO y Cuestionario de Afron- } \\
\text { tamiento al Estrés (CAE). }\end{array}$ & $\begin{array}{l}\text { El Neuroticismo correlacionó con estrategias de } \\
\text { afrontamiento pasivas y centradas en las emocio- } \\
\text { nes, consideradas no adaptativas. La extraversión, } \\
\text { amabilidad y responsabilidad se asocian con } \\
\text { afrontamiento activo y centrado en la solución de } \\
\text { problemas. }\end{array}$ \\
\hline $\begin{array}{l}\text { Ansiedad y } \\
\text { depresión }\end{array}$ & $\begin{array}{l}\text { Castellanos y } \\
\text { Guarnizo (2011) }\end{array}$ & $\begin{array}{l}\text { Escala Estrategias de Coping } \\
\text { de Chorot y Sandin y la escala } \\
\text { Zung. }\end{array}$ & $\begin{array}{l}\text { Se encontró una alta correlación negativa entre la } \\
\text { ausencia de ansiedad y la estrategia de afronta- } \\
\text { miento solución de problemas. La ansiedad severa } \\
\text { presenta una relación positiva con la estrategia de } \\
\text { afrontamiento evitación cognitiva. }\end{array}$ \\
\hline
\end{tabular}



llares (2007) Stressful Situations, Inventario de Depresión de Beck,
Sanjuán y Maga- cional, Coping Inventory for

El estilo explicativo autoensalzante se relaciona con el empleo de estrategias de afrontamiento dirigidas a la solución directa de los problemas, y que éstas, a su vez, se relacionan negativamente con el desarrollo de síntomas depresivos, y también con la no utilización de las estrategias centradas en la emoción, las cuales se asocian positivamente con la sintomatología depresiva.

\begin{tabular}{|c|c|c|c|}
\hline \multirow[t]{4}{*}{$\begin{array}{l}\text { Bienestar } \\
\text { psicológico }\end{array}$} & $\begin{array}{l}\text { Contini de Gon- } \\
\text { zález, Coronel } \\
\text { de Pace, y Levin, } \\
\text { Estevez Suedan } \\
\text { (2003) }\end{array}$ & $\begin{array}{l}\text { Escala de estrategias de } \\
\text { afrontamiento (ACS, forma } \\
\text { general) y la Escala de Bien- } \\
\text { estar Psicológico para Adoles- } \\
\text { centes (BIEPS-J) }\end{array}$ & $\begin{array}{l}\text { El afrontamiento pasivo se correlaciona con bajo } \\
\text { nivel de bienestar constituyendo factores de riesgo } \\
\text { para la salud. Las estrategias activas se asocian con } \\
\text { elevado nivel de bienestar y factores protectores de } \\
\text { la salud. }\end{array}$ \\
\hline & $\begin{array}{l}\text { Cornejo y Lucero } \\
\text { (2005) }\end{array}$ & $\begin{array}{l}\text { Escala de Bienestar Psicológi- } \\
\text { co (E.B.P.) y Cuestionario de } \\
\text { Estrategias de Afrontamiento } \\
\text { (C.S.I.) }\end{array}$ & $\begin{array}{l}\text { Se encontró que estrategias de afrontamiento } \\
\text { como la solución de problemas y la búsqueda de } \\
\text { apoyo social se asocian con bienestar psicológico. }\end{array}$ \\
\hline & $\begin{array}{l}\text { Figueroa, Con- } \\
\text { tini, Lacunza, } \\
\text { Levín y Suedan } \\
\text { (2005) }\end{array}$ & $\begin{array}{l}\text { Escala de afrontamiento para } \\
\text { adolescentes (ACS) y Escala } \\
\text { de Bienestar Psicológico para } \\
\text { adolescentes adaptación } \\
\text { Casullo (BIEPS-J) }\end{array}$ & $\begin{array}{l}\text { Existe correlación positiva entre bienestar psicoló- } \\
\text { gico y el uso de estrategias de afrontamiento que } \\
\text { implican un papel activo por parte del sujeto. Los } \\
\text { adolescentes con bajo bienestar tienden a utilizar } \\
\text { estrategias de afrontamiento del tipo pasivo ya que } \\
\text { no requieren de empeño por parte del sujeto, sino } \\
\text { una evitación del problema y el alivio de la tensión. }\end{array}$ \\
\hline & Salotti (2006) & $\begin{array}{l}\text { Escala de Estrategias de } \\
\text { Afrontamiento para adoles- } \\
\text { centes (ACS) y la Escala de } \\
\text { Bienestar Psicológico para } \\
\text { adolescentes (BIEPS-J) }\end{array}$ & $\begin{array}{l}\text { Los adolescentes que utilizan con mayor frecuen- } \\
\text { cia estrategias de afrontamiento que pertenecen } \\
\text { a un estilo dirigido a la resolución del problema o } \\
\text { en relación a los demás, presentaron un nivel de } \\
\text { bienestar psicológico significativamente mayor que } \\
\text { aquellos que utilizan estrategias de estilo impro- } \\
\text { ductivo. }\end{array}$ \\
\hline
\end{tabular}




\begin{tabular}{|c|c|c|c|}
\hline \multirow[t]{4}{*}{ Género } & $\begin{array}{l}\text { Fantin, Forenti- } \\
\text { no y Correché } \\
(2005)\end{array}$ & $\begin{array}{l}\text { Escala de Afrontamiento de } \\
\text { Frydenbergy Lewisy e inven- } \\
\text { tario de personalidad adoles- } \\
\text { cente de Millon. }\end{array}$ & $\begin{array}{l}\text { Existen diferencias en el uso de estrategias de } \\
\text { afrontamiento basadas en la condición de género. } \\
\text { Los hombres recurren a ignorar el problema y a la } \\
\text { distracción física, y las mujeres tienden a la bús- } \\
\text { queda de apoyo social, solución de problemas y la } \\
\text { preocupación. }\end{array}$ \\
\hline & $\begin{array}{l}\text { Fernández y Polo } \\
\text { (2011) }\end{array}$ & $\begin{array}{l}\text { Inventario de Estrategias de } \\
\text { Afrontamiento (CSI), Inventa- } \\
\text { rio de Estrés Académico (IEA) } \\
\text { y la Escala de Bienestar Psico- } \\
\text { lógico (EBP). }\end{array}$ & $\begin{array}{l}\text { Se encontraron diferencias en el uso de estrategias } \\
\text { de afrontamiento basadas en la condición de géne- } \\
\text { ro. Los estudiantes varones en mayor medida que } \\
\text { las mujeres, evitaron pensamientos y conductas } \\
\text { relacionadas con el acontecimiento estresante. }\end{array}$ \\
\hline & $\begin{array}{l}\text { Garaigordobil } \\
(2001)\end{array}$ & $\begin{array}{l}\text { Cuestionario de estrategias } \\
\text { cognitivas de resolución de } \\
\text { situaciones sociales. }\end{array}$ & $\begin{array}{l}\text { Se concluye que los varones utilizan afrontamiento } \\
\text { centrado en la distracción, así como estrategias } \\
\text { centradas en ignorar el problema o reservarlo para } \\
\text { sí, y las mujeres buscan más apoyo social. }\end{array}$ \\
\hline & $\begin{array}{l}\text { Martín, Lucas, y } \\
\text { Pulido (2011) }\end{array}$ & $\begin{array}{l}\text { Escala de afrontamiento para } \\
\text { Adolescentes. }\end{array}$ & $\begin{array}{l}\text { Las adolescentes utilizan estrategias centradas en } \\
\text { un estilo de afrontamiento constructivo, como con- } \\
\text { centrarse en resolver el problema o preocuparse. } \\
\text { En cuanto a los adolescentes concluyeron que uti- } \\
\text { lizan afrontamiento centrado en la distracción, así } \\
\text { como estrategias centradas en ignorar el problema } \\
\text { o reservarlo para sí. }\end{array}$ \\
\hline $\begin{array}{l}\text { Capacidad } \\
\text { intelectual }\end{array}$ & $\begin{array}{l}\text { Ramírez, Bravo y } \\
\text { Vaquero (2011) }\end{array}$ & $\begin{array}{l}\text { Cuestionario de Modos de } \\
\text { Afrontamiento (adaptado de } \\
\text { Lazarus \& Folkman, 1986) }\end{array}$ & $\begin{array}{l}\text { El uso de estrategias consideradas como funciona- } \\
\text { les y adaptativas, probablemente se relaciona con } \\
\text { recursos personales como la capacidad intelectual, } \\
\text { el nivel educativo, la solución de problemas y las } \\
\text { habilidades sociales. }\end{array}$ \\
\hline Cultural & $\begin{array}{l}\text { Frydenberg, } \\
\text { Lewis, Kennedy, } \\
\text { Ardila, Frindte y } \\
\text { Hannoun (2003) }\end{array}$ & $\begin{array}{l}\text { Escala de Afrontamiento de } \\
\text { Frydenbergy Lewis. }\end{array}$ & $\begin{array}{l}\text { Se encuentran diferencias entre países, concluyen- } \\
\text { do que los adolescentes colombianos tienden a } \\
\text { buscar pertenencia, fijarse en lo positivo, concen- } \\
\text { trarse en resolver el problema, buscar apoyo espiri- } \\
\text { tual y preocuparse. }\end{array}$ \\
\hline
\end{tabular}

Tabla 1 / Artículos que relacionan los estilos y estrategias de afrontamiento con otras variables.

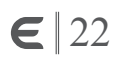


En los resultados se identificó que el instrumento utilizado con mayor frecuencia para el estudio de los estilos y estrategias de afrontamiento fue la Escala de Afrontamiento para adolescentes (ACS). Los hallazgos indican que el tipo de recurso que utiliza el estudiante puede favorecer su situación académica o por el contrario empeorarla. Por otro lado se identificaron investigaciones que resultan complementarias porque abordan la multiplicidad de variables tanto individuales como contextuales, que podrían llegar a participar en la forma en que cada estudiante afronta situaciones relacionadas con las demandas del contexto académico.

De igual forma, el conocimiento y análisis de los factores que hacen posible el manejo de la tensión es la clave de las ciencias interesadas en el bienestar y la salud del sujeto. En la investigación realizada por Figueroa, Contini, Lacunza, Levin y Suedan (2005) se consideró que el adecuado control de la tensión frente a los estresores parece determinar un afrontamiento exitoso y un concomitante estado de bienestar. Por su parte, Viñas y Caparrós (2000) constatan que las estrategias de afrontamiento pueden regular o mediatizar consecuencias negativas para la salud que trae consigo el estrés, ya que disminuye la competencia inmunológica del individuo. Además su investigación muestra que las estrategias de afrontamiento focalizadas en el problema que corresponde a afrontamiento activo de la situación están relacionadas con un mayor bienestar físico.

La adolescencia es una etapa que presenta diversas tareas que podrían convertirse es fuentes de estrés. Solís y Vidal (2006) afirman que para los adolescentes los problemas vinculados al colegio, la incertidumbre respecto al futuro, las dificultades familiares, la presión social, los problemas relacionados al uso del tiempo libre, las relaciones amorosas y los problemas en relación consigo mismos constituyen fuentes de estrés. Otras investigaciones giran en torno al cómo los adolescentes enfrentan estas demandas llevando a cabo estudios de tipo relacional en las que se concluyen correlaciones positivas entre el tipo de afrontamiento y variables como el bienestar psicológico, el nivel de adaptación, consumo de drogas y las características de personalidad (Martínez y Mikkelsen, 2009; Figueroa, Contini y Cols, 2005; Gaeta, 2009). De igual forma Viñas y Caparrós (2000), indican que las variables de personalidad pueden incidir positiva o negativamente sobre el estado de salud, tanto física como mental, de las personas. 
Otra de las perspectivas abordadas en el estudio del afrontamiento y adolescentes es la relación de tipo de afrontamiento con variables socio-demográficas como la edad, el sexo y el nivel socio-económico.

Diversas investigaciones muestran que las estrategias de afrontamiento que utiliza el sujeto pueden influir en éste, además los tipos de estrategias que implemente correlacionan con el bienestar psicológico y factores protectores de salud. Un aspecto a destacar es que el afrontamiento activo y estados de bienestar están relacionados. Sin embargo indican que no se puede señalar qué variable influye sobre la otra (Barrón, Castilla, Casullo y Verdú, 2002; Contini, Pace y cols, 2003; Figueroa, Contini y cols, 2005; Cornejo y Lucero 2005). De acuerdo con Figueroa y cols, (2005), los adolescentes con bajo bienestar tienden a utilizar estrategias de afrontamiento del tipo pasivo ya que no implican empeño por parte del sujeto, sino mas bien una evitación del problema y el alivio de la tensión. Por otro lado, Cornejo y Lucero (2005) expresan que niveles adecuados de bienestar psicológico se asocian a adecuadas estrategias de afrontamiento como la solución de problemas y búsqueda de apoyo social.

También se ha relacionado la variable adaptación al tipo de afrontamiento que desarrolle el sujeto. En este sentido, Arjona y Guerrero (2001) afirman que las estrategias de afrontamiento denominadas activas correlacionan con las habilidades sociales y con la adaptación en los sujetos. Dentro de las estrategias activas la que más utilizada es la búsqueda de soluciones relacionándose de forma positiva con la adaptación global en el sujeto. Por el contrario, las estrategias de afrontamiento pasivas (evitación), conllevan a dificultades en la adaptación global del sujeto. Es decir, aquellos sujetos de menor adaptación global tenderían a mostrar un uso mayor de estrategias de tipo pasivo, evitando el enfrentamiento directo de la situación. Así mismo, el uso del afrontamiento activo correlaciona positivamente con la mayor presencia de habilidades sociales e inteligencia emocional en los sujetos, y la adaptación se asocia de forma negativa con el uso de estrategias de afrontamiento pasivo. No obstante, Moradi, Pishva, Ehsan, Hadadi y Pouladi (2011) encontraron relación entre algunas estrategias de tipo activo, como el control físico y bajos niveles de inteligencia emocional, concluyendo que esta variable puede tener relación con estrategias de afrontamiento tanto activas como pasivas.

\section{$\boldsymbol{E} \mid 24$}


Por otro lado, Fraguela, Luengo, Romero, Villar y Sobral (2006) realizaron la investigación sobre estilos de afrontamiento y consumo de drogas, destacándose la importancia de estrategias de afrontamiento de tipo productivo que actúan como factores de protección. Por el contrario, las estrategias no productivas se dirigen a evitar los problemas, aumentando los factores de riesgo. En esta misma línea, Viñas y Caparrós (2000) encontraron que el $20.1 \%$ de los estudiantes implementaron estrategias de afrontamiento como el consumo de alcohol u otras drogas nocivas para el organismo, cuya utilización puede generar malestar físico e inducir cambios inmunológicos.

En cuanto a las variables personalidad y estilos de afrontamiento se encuentra investigaciones de tipo correlacional llevadas a cabo por Contreras y Espinoza (2009); Fantin, Forentino y Correché (2005) y Cassaretto (2010). Los resultados arrojan asociaciones importantes y significativas entre los estilos de afrontamiento y los rasgos de personalidad. Así por ejemplo, neuroticismo se asoció de forma positiva con estilo de afrontamiento llamado mal adaptativo, el cual se relaciona con menores esfuerzos por parte del sujeto para resolver el estresor asumiendo una actitud pasiva ante los problemas (Cassaretto, 2010). En contraste, la extraversión, amabilidad y responsabilidad, correlacionaron con afrontamiento activo y centrado en la solución de problemas (Contreras y Espinoza, 2009).

Los estudios también relacionan variables sociodemográficas con los diferentes estilos de afrontamiento de los jóvenes. Frydenberg y cols (2003) indican que los adolescentes emplean con mayor frecuencia un estilo de afrontamiento productivo, (esforzarse y tener éxito y concentrarse en resolver el problema) y en segundo lugar un afrontamiento no productivo y concluyen que se responde en mayor medida a través del estilo no productivo. Dentro de los aspectos relacionados con la tendencia a la utilización del estilo no productivo, se encuentran las variables género, nivel educativo y edad (Fernández, Contini y cols., 2008).

En la investigación de Barrón, Castilla, Casullo y Verdú (2002) se concluye que con la edad el repertorio de estrategias aumenta. Sin embargo, refieren que la influencia que tiene esta variable sobre las estrategias de afrontamiento no es significativa. 
En cuanto a las diferencias por género Barrón y cols. (2002) concluyen que las mujeres tienen una gama amplia de estrategias de afrontamiento en relación con los varones. El género femenino utiliza más la estrategia de apoyo en los demás, mientras que los hombres emplean estrategias como la distracción física, ignorar el problema y reservarlo para sí. Por su parte, Fantin, Forentino y Correché (2005) encontraron que las mujeres tienden más a la búsqueda de apoyo social, solución de problemas, preocupación, y los varones recurren a ignorar el problema y a la distracción física, lo que hace pensar en evidentes diferencias significativas basadas en la condición de género. Fraguela y cols (2006) indican que las adolescentes se apoyan en los demás para la solución de los problemas, mientras que los hombres tienden a despreocuparse e intentar resolver las situaciones por sí mismos. Viñas y Caparrós (2000) refieren que mujeres que obtienen puntuaciones altas en afrontamiento de las emociones autoinforman mayores molestias fisiológicas durante el periodo menstrual.

El estudio sobre estrategias de afrontamiento pone de manifiesto la relación entre esta variable y con, satisfacción con la vida, bienestar psicológico y nivel de adaptación a diferentes contextos en los adolescentes. Las estrategias que utilice el sujeto según lo planteado por Lazarus y Folkman (1986) no dependen de las situaciones en sí misma, sino la interpretación que el sujeto realiza de tales situaciones, por lo cual se asume un papel activo por parte del sujeto para poder enfrentar las situaciones de forma efectiva.

En cuanto a bienestar psicológico, Castro, Solano y Casullo (2000) infieren que es una variable que establece diferencias significativas en los estilos y estrategias de afrontamiento que utilizan los jóvenes; el tipo de estrategias de afrontamiento que utilice el sujeto se asocian con bienestar psicológico y factores protectores de salud. Así, cuando se utilizan estrategias orientadas a resolver el problema, el bienestar psicológico que percibe la persona es alto, y los sujetos que utilizan estrategias de afrontamiento no productivo como ignorar el problema o autoinculparse perciben un bajo bienestar psicológico.

Los resultados de las investigaciones proponen el inicio de programas enfocados en la promoción de estilos de vida saludable para la adolescencia temprana, orientados a la comprensión de las diferencias individuales respecto a la forma en la que los jóvenes afrontan las demandas del ambiente.

\section{E $\| 26$}




\subsection{Rendimiento Académico}

En esta categoría se encontraron 12 artículos con diferentes diseños metodológicos, de corte relacional, documental, correlacional y no experimental, entre otros. Estas investigaciones determinan que la comprensión del rendimiento académico es multicausal. En la siguiente tabla se ilustra la información relevante frente a los estudios realizados sobre rendimiento académico.

\begin{tabular}{|c|c|c|c|}
\hline Categoría & Autores & Instrumentos & Resultados \\
\hline & $\begin{array}{l}\text { Aldana, Pérez \& } \\
\text { Rodríguez (2010) }\end{array}$ & $\begin{array}{l}\text { Detección, procesamiento y aná- } \\
\text { lisis de material bibliográfico }\end{array}$ & $\begin{array}{l}\text { Se concluye que el bajo rendimiento académico tiene } \\
\text { múltiples causas teniendo presente la responsabilidad de } \\
\text { los diferentes actores: estudiantes, institución y directi- } \\
\text { vos y docentes. }\end{array}$ \\
\hline & \multicolumn{2}{|l|}{$\begin{array}{l}\text { Caballero, Abello } \\
\text { \& Palacio (2007) }\end{array}$} & $\begin{array}{l}\text { Los estudiantes con bajo rendimiento académico } \\
\text { tienden a percibir baja autoeficacia }\end{array}$ \\
\hline & $\begin{array}{l}\text { Carvajal, Mosquera } \\
\text { \& Artamonova } \\
\text { (2009) }\end{array}$ & $\begin{array}{l}\text { Modelo de regresión logística } \\
\text { múltiple }\end{array}$ & $\begin{array}{l}\text { Las variables sociodemográficas, así como factores de } \\
\text { riesgo en salud no tienen relación significativa con el } \\
\text { rendimiento académico en matemáticas, sin embargo se } \\
\text { encuentra asociación entre el resultado del examen ICFES } \\
\text { y bajo rendimiento en la misma área. }\end{array}$ \\
\hline & \multicolumn{2}{|l|}{$\begin{array}{l}\text { Hernández, } \\
\text { Coronado, } \\
\text { Araujo \& Cerezo } \\
\text { (2008) }\end{array}$} & $\begin{array}{l}\text { Los estudiantes con bajos promedios académicos } \\
\text { presentan altos niveles de ansiedad. }\end{array}$ \\
\hline & $\begin{array}{l}\text { Rodríguez } \\
\text { (2012). }\end{array}$ & & $\begin{array}{l}\text { Se encontró que la carencia de competencias aca- } \\
\text { démicas en los estudiantes es un asunto que com- } \\
\text { pete a diversos profesionales, pero se enfatiza en el } \\
\text { papel que deben asumir las directivas instituciona- } \\
\text { les para resolver el problema. }\end{array}$ \\
\hline & $\begin{array}{l}\text { Saldaña \& Barriga } \\
\text { (2010) }\end{array}$ & Análisis de eventos históricos & $\begin{array}{l}\text { Concluyen que la probabilidad de sufrir un evento de } \\
\text { deserción se produce mayoritariamente en primer y } \\
\text { segundo año con un porcentaje del } 50 \% \text { y al aumentar el } \\
\text { promedio de notas cada semestre se disminuyen estos } \\
\text { índices. }\end{array}$ \\
\hline
\end{tabular}




\begin{tabular}{|c|c|c|}
\hline $\begin{array}{l}\text { Sánchez \& Pirela } \\
\text { (2009) }\end{array}$ & $\begin{array}{l}\text { MLP (Inventario de motivación al } \\
\text { logro) MAFI (Inventario de afilia- } \\
\text { ción) (MPS) Inventario de poder }\end{array}$ & $\begin{array}{l}\text { El rendimiento académico tiene una correlación positiva } \\
\text { con la motivación al logro, negativa con motivación de } \\
\text { afiliación y no se relaciona con la motivación al poder. }\end{array}$ \\
\hline $\begin{array}{l}\text { Tejedor, García \& } \\
\text { Muñoz (2007) }\end{array}$ & Cuestionario estructurado & $\begin{array}{l}\text { Se encontró acuerdo entre los actores en que el bajo } \\
\text { rendimiento académico depende de muchos actores: los } \\
\text { estudiantes, la institución y los docentes. Se plantean } \\
\text { estrategias para superarlo destacándose la motivación al } \\
\text { estudiantado, la orientación profesional y la capacitación } \\
\text { permanente por parte de los docentes. }\end{array}$ \\
\hline Tonconi (2010) & & $\begin{array}{l}\text { Los estudiantes con buen rendimiento académico } \\
\text { cuentan con mayores posibilidades de culminar } \\
\text { satisfactoriamente sus estudios de la carrera, } \\
\text { mientras que, los estudiantes con bajo rendimiento } \\
\text { tienden a perder las materias, lo cual incrementa el } \\
\text { nivel de la deserción de los estudiantes. }\end{array}$ \\
\hline Yengle (2009) & & $\begin{array}{l}\text { Se encontró que la mayor frecuencia de deserción } \\
\text { estudiantil se produce en los primeros tres o cuatro } \\
\text { semestres o ciclos de estudio, principalmente en el } \\
\text { primer semestre. La dificultad de adaptación está } \\
\text { relacionada con la interacción de los estudiantes } \\
\text { con sus compañeros de estudio. }\end{array}$ \\
\hline $\begin{array}{l}\text { Villalba \& Barra- } \\
\text { gán (2008) }\end{array}$ & & $\begin{array}{l}\text { Existe correlación positiva entre el rendimiento } \\
\text { académico en la educación media y el que se obtie- } \\
\text { ne en la universidad }\end{array}$ \\
\hline
\end{tabular}

Tabla 2 / Información relevante frente a los estudios realizados sobre rendimiento académico.

\subsubsection{Variables Relacionadas con el Rendimiento Académico}

La comprensión de la problemática parte de la definición de rendimiento académico y de la comprensión de características inherentes al ambiente universitario. Según Aldana, Pérez y Rodríguez (2010) el rendimiento académico es un fenómeno multifactorial en el que se identifican las dimensiones como: características del estudiante, de la institución, expresiones del rendimiento y trayectoria estudiantil. En esta misma línea Tonconi (2010) refiere que el bajo rendimiento académico es considerado como multifactorial y como una problemática que

\section{$\boldsymbol{\epsilon} \| 28$}


se debe atender de forma inmediata ya que se mantiene a pesar de las estrategias que se han utilizado para contrarrestarlo.

De acuerdo con lo anterior, Enríquez (2008) y Tejedor y cols (2007), refieren que el bajo rendimiento académico en estudiantes universitarios oscilan entre el $26,5 \%$ y $27 \%$. Asimismo, Tejedor y cols. (2007); Saldaña y Barriga (2010) y Yengle (2009) refieren que el fracaso académico (abandono) se concentra en los primeros cursos, acumulándose el $90 \%$ de los alumnos desertores entre 10 y $2 \circ$ grado de carrera. Esta tasa está en consonancia con los datos obtenidos en el seguimiento de cohortes en otras Universidades españolas y pone de manifiesto, la importancia del fracaso académico en ambientes universitarios (Tejedor y cols, 2007).

En cuanto a las causas de la problemática Tejedor y cols. (2007) indican que se pueden clasificar en tres categorías: institucionales, relacionadas con el profesor y relacionadas con el alumno. Por su parte Tonconi (2010), infiere que no existe un conjunto definido de variables explicativas, sino que éstas dependen del contexto socioeconómico y cultural en el cual se lleve a cabo cada investigación. Por ejemplo, se tienen en cuenta variables como las políticas universitarias, el diseño de cada programa, la importancia que da cada institución en la asistencia a clase y/o el número de créditos para cada asignatura.

Por su parte, Aldana, Pérez y Rodríguez (2010) concluyen que tanto la institución, los estudiantes y los profesores deben compartir responsabilidades para contrarrestar el bajo rendimiento académico. Así mismo consideran que esta es una situación que se repite año tras año, y que no ha sido posible articular programas o cursos de acción realmente efectivos para enfrentarla. La tendencia en las instituciones de educación superior para afrontar el bajo rendimiento académico se centra en estrategias institucionales como la creación de espacios y acceso a los recursos para estimular el trabajo académico, capacitaciones para los docentes, tutorías académicas, entre otras. (Tejedor y cols 2007). Pero, se considera necesario orientar los esfuerzos para el fortalecimiento de habilidades del estudiante como el uso de adecuadas estrategias de afrontamiento.

La adaptación escolar es otro factor importante que promueve el buen rendimiento académico, Prior y cols. (2011); Londoño (2009) y Yengle (2009) evidencian la importancia de las 
habilidades sociales y el apoyo social percibido en la adaptación y ajuste de los alumnos al nuevo medio académico, lo que facilita la integración escolar y la consecución de objetivos académicos. La variable adaptación al contexto universitario ha adquirido principal importancia, esta se evidencia en las conclusiones de los estudios hechos por los autores referidos, quienes señalan que existe relación positiva entre la variable adaptación y el éxito académico.

Una de las consecuencias del bajo rendimiento académico es la deserción estudiantil. Según, Saldaña y Barriga (2010) y Tonconi (2010) existen diferentes factores que la promueven, en cuanto a género refieren que las mujeres desertarían más que los hombres en carreras como las ingenierías y lo asocian con el hecho de que los hombres tienen mayor puntaje en las pruebas de selección universitaria en matemáticas y mejores promedios de nota semestral en la carrera. A mejor promedio de notas cada semestre, menos probabilidad de registrar un evento de deserción al semestre siguiente. Otro factor determinante es la ayuda financiera entregada al alumno, la cual es fundamental para su integración universitaria, compromiso con la institución y continuación de estudios.

Se reporta en la literatura el análisis de variables como las competencias académicas específicas en estudiantes universitarios con enfermedades crónicas y sin ellas. Se concluye que los participantes sin enfermedades crónicas son en general más competentes que los que tienen alguna enfermedad. Estos estudiantes cumplen con más frecuencia con las diversas demandas académicas, no reaccionan con estrés con tanta frecuencia y no valoran las situaciones presentadas en los instrumentos como amenazantes (Rodríguez, 2012).

El análisis hecho por Tejedor y cols. (2007) da como resultado el planteamiento de estrategias que deben abordar cada uno de los actores en la superación del bajo rendimiento académico. Los autores perciben la necesidad de mejorar los procesos de orientación profesional y vocacional, los hábitos y técnicas de estudio y las actitudes de responsabilidad por parte de los estudiantes. Así mismo, sugieren que los docentes orienten y apoyen al alumnado en las dificultades de aprendizaje y que las instituciones educativas brinden estrategias para elevar el nivel de conocimientos, favorecer las actividades culturales y de estudio; y también crear espacios y acceso a los recursos para estimular el trabajo académico. Se considera importante aportar al análisis de Tejedor la inclusión de variables de tipo psicológico, fundamentales para lograr una integración de las estrategias para superar el bajo rendimiento académico.

$\boldsymbol{\epsilon} \| 30$ 


\subsection{Estilos y Estrategias de afrontamiento y rendimiento académico}

En esta categoría se encontraron 10 artículos, con diferentes diseños metodológicos, de corte relacional, documental, correlacional y no experimental, entre otros. Estas investigaciones determinan que la comprensión del rendimiento académico es multicausal. En la tabla 3 se visualiza las estrategias de Afrontamiento y Rendimiento Académico.

\begin{tabular}{|c|c|c|}
\hline Autores & Instrumentos & Resultados \\
\hline $\begin{array}{l}\text { Ballester, March \& } \\
\text { Irte (2006) }\end{array}$ & $\begin{array}{l}\text { Cuestionario de autocon- } \\
\text { cepto y estilos de afron- } \\
\text { tamiento, cuestionario de } \\
\text { motivación, satisfacción } \\
\text { con los estudios y prácticas } \\
\text { educativas }\end{array}$ & $\begin{array}{l}\text { Los alumnos que sintieron que sus capacidades intelectuales y sus } \\
\text { características personales fueron valoradas por sus compañeros, } \\
\text { profesores y por ellos mismos, seleccionaron las estrategias de } \\
\text { relación interpersonal más adecuadas formando estilos de afron- } \\
\text { tamiento más eficaces. }\end{array}$ \\
\hline $\begin{array}{l}\text { De la Cruz y Monta- } \\
\text { ña (2011) }\end{array}$ & $\begin{array}{l}\text { Inventario Alemán de ansie- } \\
\text { dad frente a los exámenes, } \\
\text { G-TAl-, evocando alguna } \\
\text { situación de examen }\end{array}$ & $\begin{array}{l}\text { Se evidenció que cuando los estudiantes tienen altos niveles de } \\
\text { ansiedad, utilizan más la estrategia de evitación- escape, (presente } \\
\text { principalmente en los estudiantes de primer año), de igual forma } \\
\text { a menor nivel de ansiedad, se utiliza con mayor frecuencia la solu- } \\
\text { ción de problemas. }\end{array}$ \\
\hline $\begin{array}{l}\text { Fernández y cols } \\
\text { (2008) }\end{array}$ & Cuestionario de estrategias & $\begin{array}{l}\text { Los estudiantes de sexo masculino apelan a su bagaje de afron- } \\
\text { tamiento en forma más variada y plástica, no evidencian el uso } \\
\text { prevalente de ninguna herramienta comportamental o cognitiva } \\
\text { en particular. }\end{array}$ \\
\hline Martínez (2010) & $\begin{array}{l}\text { Escala de Afrontamiento } \\
\text { para Adolescentes (Fryden- } \\
\text { berg y Lewis, 1996). }\end{array}$ & $\begin{array}{l}\text { Se encontró que los alumnos que se esfuerzan y se comprometen } \\
\text { para afrontar el estrés académico tienden a obtener mejor rendi- } \\
\text { miento escolar. Por el contrario, los estudiantes universitarios que } \\
\text { no afrontan los problemas o que los ignoran, tienden a obtener } \\
\text { deficientes resultados académicos. }\end{array}$ \\
\hline
\end{tabular}


Frydenberg, Lewis, Kennedy, Ardila, Frindte \& Hannoun Adolescent Coping Scale. (2003)
Los adolescentes emplearon un estilo de afrontamiento productivo, específicamente las estrategias de: Esforzarse y tener éxito y Concentrarse en resolver el problema; y en segundo lugar un afrontamiento no Productivo. Sin embargo existen diferencias significativas entre países, como: Colombia, Austria, Alemania y Palestina. La muestra colombiana mostró tendencia a: buscar pertenencia, fijarse en lo positivo, acción social, concentrarse en resolver el problema, buscar apoyo espiritual y preocuparse.

\begin{tabular}{|c|c|c|}
\hline $\begin{array}{l}\text { Furlan, Sánchez, } \\
\text { Heredia, Piemonte- } \\
\text { si, Illbele y Martínez } \\
\text { (2012) }\end{array}$ & $\begin{array}{l}\text { Adaptación argentina de } \\
\text { la Tuckman Procrastina- } \\
\text { tion Scale. Adaptación del } \\
\text { inventario German Test } \\
\text { Anxiety Inventory. Adapta- } \\
\text { ción abreviada de la escala } \\
\text { Self-Efficacy for Learning } \\
\text { Form. }\end{array}$ & $\begin{array}{l}\text { Se asoció en forma positiva, la preocupación con las estrategias } \\
\text { de repetición y regulación del esfuerzo y con estilos más o menos } \\
\text { adaptativos de afrontamiento. Inversamente, la falta de confianza } \\
\text { y la interferencia se relacionan negativamente con estrategias de } \\
\text { aprendizaje reflexivo y crítico. }\end{array}$ \\
\hline $\begin{array}{l}\text { Piemontesi, Here- } \\
\text { dia, Furlan, Sánchez } \\
\text { y Martínez (2001) }\end{array}$ & $\begin{array}{l}\text { Adaptación preliminar del } \\
\text { R-COPE. Adaptación argen- } \\
\text { tina del inventario alemán } \\
\text { de ansiedad frente a los } \\
\text { exámenes (GTAI-A). }\end{array}$ & $\begin{array}{l}\text { Encontraron que los estudiantes más ansiosos se caracterizaron } \\
\text { por los estilos de autoculpa y rumiación autofocalizada y los me- } \\
\text { nos ansiosos por aproximación y acomodación. Las correlaciones } \\
\text { parciales entre cada dimensión y el afrontamiento indicaron que la } \\
\text { preocupación se asocia positivamente a la aproximación; un estilo } \\
\text { de afrontamiento más adaptativo que favorece la preparación y } \\
\text { el estudio, en tanto que la interferencia y la falta de confianza se } \\
\text { vinculan a estilos más disfuncionales. }\end{array}$ \\
\hline Palacio y cols (2012) & $\begin{array}{l}\text { MBI-SS y a la Escala de Es- } \\
\text { trategias de Afrontamiento- } \\
\text { Modificada }\end{array}$ & $\begin{array}{l}\text { Dentro de las estrategias de afrontamiento utilizadas con mayor } \\
\text { frecuencia por los estudiantes, se hallaron: solución de problemas, } \\
\text { la cual se asocia con resultados más favorables en el promedio } \\
\text { académico, siguiendo el orden están la evitación emocional, apoyo } \\
\text { Social, espera y religión. }\end{array}$ \\
\hline
\end{tabular}


validación la BCS (Escala del afrontamiento al aburrimiento), así como en hallar

Tze y cols (2013) perfiles de afrontamiento efectivos a la hora de disminuir el aburrimiento y aumentar el éxito académico.
Se encontraron dos perfiles de afrontamiento: uno crítico, consistente en actitudes novedosas en pro de mejorar el rendimiento académico; el segundo es de tipo reevaluador, dirigido a concentrarse en las propias emociones relacionadas con el estudio, descuidando el interés por el propio desempeño. Se encontró que el primero de estos perfiles es más efectivo y no se encontraron diferencias significativas en cuanto al mejoramiento del rendimiento académico.
Veloso, Caqueo, Muñoz y Villegas (2010)
Escala de Afrontamiento para Adolescentes.
Concluyen que las instituciones educativas de tipo particular subvencionado disponen de una mayor gama de estrategias focalizadas en las emociones y en la reducción del problema, lo cual puede deberse a su contexto psicosocial.

Tabla 3 / Estrategias de Afrontamiento y Rendimiento Académico.

La última perspectiva de investigación del presente estudio se centra en el análisis de la relación de los estilos y estrategias de afrontamiento y el rendimiento académico. En este sentido, el estudio realizado por Palacio y cols. (2012) ofrece un valioso aporte teórico, ya que indaga la relación entre las variables objeto del presente artículo y el burnout académico. Los resultados mostraron que la mayoría de los estudiantes presentaban bajos niveles de estrés académico. Dentro de las estrategias de afrontamiento utilizadas con mayor frecuencia por los estudiantes se identificó la solución de problemas, la cual se asocia con resultados más favorables en el promedio. Siguiendo el orden están la evitación emocional, el apoyo social y la religión (Palacio y cols. 2012). De igual forma, Hoyoung y Cols (2011) concluyen que altos niveles de estrés son asociados con las respuestas de tipo evitativo, así como la tendencia a la búsqueda de la novedad.

En esta misma línea Martínez (2010) relacionó las estrategias de afrontamiento ante el estrés y el rendimiento académico. Los resultados demuestran que los alumnos que se esfuerzan y se comprometen para afrontar el estrés académico tienden a obtener mejor rendimiento. De igual forma las respuestas consistentes en esforzarse, tener éxito y resolver el problema, parecen estar dirigidas a centrar más al estudiante en su labor.

Por otra parte, De la Cruz y Montaña (2011) realizaron un estudio con el objetivo de conocer cuáles son las estrategias más utilizadas en el primer y último año de estudio, frente a situaciones de 
ansiedad en escenarios de examen. En el análisis de la información, se encontró que las mujeres recurrieron más a estrategias de solución de problemas, de apoyo social, mostraron falta de confianza y mayor preocupación y emocionalidad. Por el contrario, los hombres no mostraron una respuesta preferida en el afrontamiento, presentaron una tendencia a implementar niveles de preocupación e interferencia.

Otro resultado importante estuvo relacionado con el año de inicio, ya que evidenciaron que los estudiantes de primer año, no eligieron un estilo de afrontamiento específico; mostraron falta de confianza y preocupación, mientras que los alumnos de quinto año, aunque también presentaron falta de confianza y preocupación, utilizaron conductas relacionadas con la solución de problemas.

En dicho estudio se pudo evidenciar que cuando los estudiantes tienen altos niveles de ansiedad, utilizan más la estrategia de evitación - escape, (presente principalmente en los estudiantes de primer año). Por el contrario, ante un menor nivel de ansiedad, se utiliza más la estrategia de solución de problemas.

Por su parte, Tze y cols. (2013) realizaron un estudio sobre las estrategias de afrontamiento respecto al aburrimiento académico en estudiantes universitarios. El estudio arrojó como resultado dos perfiles de afrontamiento: por un lado uno crítico, consistente en la manifestación de sus sentimientos y la búsqueda de actividades novedosas asociadas con el estudio en pro de mejorar el rendimiento académico; por otro lado es el re-evaluador, consistente en concentrarse en las propias emociones relacionadas con el estudio, descuidando el interés por el propio desempeño. No se encontraron diferencias significativas en cuanto al mejoramiento del rendimiento académico en los dos perfiles descritos.

Piemontesi y cols. (2001) exploraron las diferencias en los jóvenes, respecto a los estilos de afrontamiento con baja, moderada y alta ansiedad ante los exámenes. El análisis de resultados muestra que los estudiantes más ansiosos se caracterizan por los estilos de autoculpa y rumiación autofocalizada y los menos ansiosos por aproximación y acomodación.

En una investigación posterior Furlan y cols. (2012) plantearon el mismo objetivo, encontrando que los estudiantes más ansiosos, presentaron mayor tendencia a la auto culpabilización y la

$\boldsymbol{E} \| 34$ 
rumiación auto-focalizada. Los menos ansiosos se orientaron hacia el problema buscando su resolución o su aceptación y re-evaluación en términos más positivos.

De acuerdo a Piemontesi y Heredia (2009) la dimensión “preocupación" conlleva un mayor uso de estrategias tendientes a aprovechar el tiempo, administrar el esfuerzo, fijar conocimientos y resolver dudas. Esta tendencia a la movilización de recursos se había observado en estudios previos, donde esta variable se relacionó con la implementación de conductas orientadas al problema y una mayor autoeficacia para la autorregulación del aprendizaje.

Veloso, Caqueo, Muñoz y Villegas (2010) realizaron un estudio que profundiza el cómo el contexto educativo interviene en las Estrategias de Afrontamiento utilizadas por los Adolescentes, encontrando que las implementadas principalmente fueron: buscar diversión relajante, preocuparse, esforzarse, tener éxito y fijarse en lo positivo, lo cual puede deberse al contexto psicosocial.

\section{Discusión}

Se encontró un número importante de estudios sobre estilos y estrategias de afrontamiento y su relación con otras variables. De igual forma, se identificó una amplia gama de investigaciones interesadas en el análisis de rendimiento académico. Sin embargo, se evidenció la baja tendencia a relacionar las dos variables objeto del presente estudio: los estilos y estrategias de afrontamiento y rendimiento académico.

Las investigaciones relacionadas con estilos y estrategias de afrontamiento coinciden en que la población en general evalúa muchas de las situaciones de la vida cotidiana y específicamente el contexto universitario como estresantes de acuerdo a como lo señalan Fernández y Polo (2011) y Piemontesi y cols. (2001), razón por la cual el estudio sobre afrontamiento toma importancia al ser considerado como un mecanismo para responder a estas situaciones de demanda que le permitiría al estudiante avanzar en su proyecto académico.

De acuerdo a lo anterior, diferentes autores como Arjona y cols. (2001); Morales y cols. (2010) coinciden en que el uso de estrategias de afrontamiento de tipo activo o centrado en la solución del problema se relaciona con variables como alto bienestar psicológico y satisfacción con la vida, un buen nivel de adaptación, inteligencia emocional y autorregulación. Por el contrario el uso de estrategias de tipo pasivo correlacionan con altos niveles de ansiedad, estrés y depresión. 
En referencia al género, autores como Fantin y cols. (2005) Garaigordobil (2001) Fernández y cols. (2011) Martin y cols. (2011) diferencian las estrategias utilizadas por hombres y mujeres: el sexo femenino utiliza más la estrategia de apoyo social, solución de problemas y preocupación; el género masculino implementa con mayor frecuencia respuestas como la distracción física, ignorar el problema, despreocuparse y resolución de situaciones por sí mismo.

En cuanto a la variable rendimiento académico, autores como Aldana y cols. (2010) Tejedor y cols. (2007) coinciden en considerarlo multifactorial, donde intervienen las áreas académica, social, familiar, personal y económica. Los artículos consultados permiten inferir la relación directa entre bajo rendimiento académico y altos niveles de deserción en forma incidente durante los primeros semestres. Tejedor y cols. (2007) infieren que esta situación presenta una tendencia a mantenerse pese a las diferentes estrategias que se puedan generar para minimizarlo, como son: la implementación de políticas institucionales, capacitación docente, departamento de bienestar universitario, entre otras. Por esta razón es prioritario trabajar en la creación o fortalecimiento de métodos eficaces que favorezcan el rendimiento académico en contextos universitarios.

El abordaje del rendimiento académico conlleva la relación de diversos factores: a nivel institucional (los directivos y docentes) y a nivel personal, (aspectos familiares, sociales, culturales y económicos). Lo anterior involucra una mirada holística como lo describen los autores Rodríguez (2012) y Aldana y cols (2010) los cuales plantean una relación entre los diferentes actores co-responsables y el fracaso académico. Sin embargo, un aspecto que resulta relevante considerar para futuras intervenciones que pretendan contribuir al rendimiento académico es la particularidad del estudiante en el uso de las estrategias de afrontamiento, sin descuidar el contexto en el cual interactúa, en tanto que puede consolidarse como una red de apoyo o un agente que lo vulnere.

En este sentido, las investigaciones consultadas concuerdan en la necesidad de mejorar los procesos de orientación profesional y vocacional, los hábitos y técnicas de estudio y las actitudes de responsabilidad, esfuerzo y auto-exigencia por parte de los estudiantes, lo que sugiere la realización de futuras investigaciones dirigidas a identificar variables psicológicas, que estén asociadas al bajo rendimiento, para así lograr un abordaje integral de la problemática.

\section{$\boldsymbol{E} \mid 36$}


De igual forma, se podrían llevar a cabo futuros estudios de las estrategias de afrontamiento implementadas por diferentes grupos de estudiantes con miras a comparar las estrategias utilizadas en nuestro contexto y las reportadas en investigaciones realizadas en otras universidades, lo anterior con el fin de caracterizar la población e identificar las posibles variables que estén influyendo en las diferencias.

Finalmente, los resultados de las investigaciones proponen el inicio de programas enfocados en la promoción de estilos de vida saludable para la adolescencia temprana, orientados a la comprensión de las diferencias individuales respecto a la forma en la que los jóvenes afrontan las demandas del ambiente, especialmente en el inicio de su vida universitaria, proyecto que resulta trascendente y requiere de estrategias de afrontamiento encaminadas a resolver los retos que implica dicho proyecto.

\section{Conclusiones}

Los estudios realizados permiten conocer las estrategias de afrontamiento utilizadas por los estudiantes de educación superior que relaciona con un adecuado desempeño académico dando respuesta así al objetivo planteado en la presente investigación. Se encuentra que los jóvenes que utilizan estrategias enfocadas en la solución acertada de la situación estresante tienen mayor probabilidad de obtener un buen rendimiento académico. De igual forma, aquellos que hacen uso de las estrategias como la religión, el esfuerzo y el éxito logran responder a las exigencias académicas.

De acuerdo con lo reportado por los autores, el apoyo social constituye una variable relevante para los jóvenes, la percepción de ser aceptado en un grupo de pares, compañeros de semestre y el apoyo de su familia es importante para el logro de las metas propuestas a nivel académico.

El análisis de investigaciones que tenían por objeto relacionar las variables estilos y estrategias de afrontamiento y rendimiento académico, permitió identificar las conductas que favorecen el rendimiento académico como: la solución de problemas, esforzarse y tener éxito. Por el contrario, las respuestas de tipo evitativo y escape, así como la tendencia a la búsqueda de la novedad correlacionaron significativamente con altos niveles de estrés, ansiedad y bajo rendimiento académico. Los estudiantes más ansiosos se caracterizaron por los estilos de autoculpa y rumiación 
autofocalizada y los menos ansiosos por aproximación y acomodación, la dimensión preocupación conlleva un mayor uso de estrategias tendientes a aprovechar el tiempo, administrar el esfuerzo, fijar conocimientos y resolver dudas.

Por último, cabe resaltar que los jóvenes universitarios que presentaron preocupación ante su situación académica implementaron sus estilos y estrategias de tipo activo, evidenciando un papel más dinámico dentro de este proceso formativo. De igual manera el uso de este tipo de estrategias se relaciona con la ubicación semestral, lo cual sugiere que en semestres avanzados se tienda a utilizar estrategias de tipo activo y se mejore su rendimiento.

\section{Notas de los autores y agradecimientos}

1. Resultado parcial de la investigación “Efectividad Del Programa De Intervención Psicológica EAPRA En Estilos Y Estrategias De Afrontamiento Frente Al Desempeño Académico En Los Estudiantes De La Universidad De Boyacá Sede Sogamoso", Grupo de investigación ETHOS, Facultad de Ciencias Humanas y de la Educación, Universidad de Boyacá.

Los autores del presente artículo agradecen a los docentes Ps. Iván Leonardo Otálora y Ps. Julio Fernando Acosta Muñoz por la asesoría metodológica y corrección de estilo.

\section{Referencias}

Aldana, K., Pérez, R., \& Rodríguez, M. (2010). Visión del desempeño académico estudiantil en la Universidad Centro Occidental Lisandro Alvarado. Revista de Investigación Científica, 3(24), 5-22. Recuperado de http://www.redalyc.org/pdf/880/88017685002.pdf

Arjona, J., \& Guerrero, S. (2001). Un estudio sobre los estilos y las estrategias de afrontamiento y su relación con la variable adaptación. Psicología Científica, 1, 1-13. Recuperado de http://ww2.educarchile.cl/UserFiles/P0001/File/psicologiapdf-182-un-estudio-sobre-los-estilos-y-las-estrategias-de-afrontamiento-y-su-relacion-co.pdf

Ballester, L., March, M., \& Irte, C. (2006). Auto concepto, estilos de afrontamiento y conducta del alumnado. Aposta. Revista de Ciencias Sociales, 27, 1-22. Recuperado de http://www.apostadigital.com/revistav3/ hemeroteca/brage.pdf 
Barrón, R., Castilla, I., Casullo, M., \& Verdú, J. (2002). Relación entre estilos y estrategias de afrontamiento y bienestar psicológico en adolescentes. Psicothema, 14(2), 363-368. Recuperado de http://www.unioviedo.net/reunido/index.php/PST/article/download/8028/7892.

Casado, F. (2002). Modelo de afrontamiento de Lazarus como heurístico de las intervenciones psicoterapéuticas. Equipo de Salud Mental Alcalá-Dos Hermanas, 20, 1-10. Recuperado de http://www.cop.es/delegaci/ andocci/files/contenidos/vol20_3_5.pdf.

Cassaretto, M. (2010). Relaciones entre la personalidad y el afrontamiento en estudiantes preuniversitarios. Revista Vanguardia Psicológica, 1(2), 202-225. Recuperado de http://umb.edu.co:82/revp/index.php/ vanguardiapsicologica/article/download/28/143

Castaño, E., \& León del Barco, B. (2010). Estrategias de afrontamiento del estrés y estilos de conducta interpersonal. International Journal of Psychology and Psychological Therapy, 10 (2), 245-257. Recuperado de http://www.ijpsy.com/volumen10/num2/260/estrategias-de-afrontamiento-del-estres-ES.pdf

Castellanos, M. T., Guarnizo, C. A., Salamanca, Y., (2011). Relación entre niveles de ansiedad y estrategias de afrontamiento en practicantes de psicología de una universidad colombiana. International Journal of Psychological Research, 4(1), 50-57. Recuperado de http://www.redalyc.org/pdf/2990/299022819007.pdf

Contreras, T., \& Espinoza, M. (2009). Personalidad y afrontamiento en estudiantes Universitarios. Revista Universitas Psychologica, 8(2), 311-322. Recuperado de http://www.redalyc.org/articulo.oa?id=64712165003

Cornejo, M., \& Lucero M. C. (2005) Preocupaciones vitales en estudiantes universitarios relacionado con bienestar psicológico y modalidades de afrontamiento. Fundamentos en Humanidades, 6(12), 143-153. Recuperado de http://www.redalyc.org/pdf/184/18412608.pdf

De la Cruz, J., \& Montaña, L. (2011). Ansiedad en situaciones de examen y estrategias de afrontamiento en alumnos universitarios de 1 y 5 año. Tesis sin publicación, Universidad Abierta interamericana, Argentina. Recuperado de http://imgbiblio.vaneduc.edu.ar/fulltext/files/TC104092.pdf

Fantin, M., Forentino, M., \& Correché, M. (2005). Estilos de personalidad y estrategias de afrontamiento en adolescentes de una escuela privada de San Luis. Fundamentos en Humanidades, 7(11), 159-176. Recuperado de http://www.redalyc.org/articulo.oa?id=18411609 
Fernández, J., \& Polo, S. (2011). Afrontamiento, estrés y bienestar psicológico en estudiantes de educación social de nuevo ingreso. EduPsykhé, 10(2), 177-192. Recuperado de http://dialnet.unirioja.es/servlet/articulo?codigo $=3896794$

Fernández, M., Contini, N., Ongarato, P., Saavedra, E., \& Iglesia, G. (2008). Estrategias de afrontamiento frente a problemas académicos. Universidad de Buenos Aires, Universidad Nacional de Tucumán. Disponible en www.aidep.org/uba/Bibliografia/afrproblacad.pdf

Fernández, M., \& Manga, R. (2009). Estrés percibido, estrategias de afrontamiento y sentido de coherencia en estudiantes de enfermería: su asociación con salud psicológica y estabilidad emocional. Tesis doctoral, Universidad de León. Recuperado de https://buleria.unileon.es/bitstream/handle/10612/902/2009FERN\%C3\%81NDEZ\%20MART\%C3\%8DNEZ,\%20MAR\%C3\%8DA\%20ELENA.pdf?sequence $=1$

Figueroa, M., Y Cohen (s,f). Estrategias y estilos de afrontamiento del estrés en adolescentes. Capítulo II. Tomado de Casullo, M. M. y Fernández Liporace (2001). Adaptado por Contini, Figueroa, Cohen Imach y Coronel (2003).

Figueroa, M., Contini, N., Lacunza, A., Levín, M., \& Suedan, A. (2005). Estrategias de afrontamiento y su relación con el nivel de bienestar psicológico. Un estudio con adolescentes de nivel socioeconómico bajo de Tucumán (Argentina). Anales de psicología, 21(1), 66-72. Recuperado de http://revistas.um.es/analesps/article/view/27171

Fraguela, J., Luengo, A., Romero, E., Villar, P., \& Sobral, J. (2006). Estrategias de afrontamiento en el inicio de la adolescencia y su relación con el consumo de drogas y la conducta problemática. International journal of clinical and health psychology, 6 (3), 581-597. Recuperado de http://www.redalyc.org/articulo. oa?id=33760305

Frydenberg, E., Lewis, R., Kennedy, G., Ardila, R., Frindte, W., \& Hannoun, R. (2003). Coping with concerns: An exploratory comparison of Australian, Colombian, German and Palestinian adolescents. Journal of Youth and Adolescence, 32, 59-66. Recuperado de http://www.researchgate.net/publication/226471030_ Coping_with_Concerns_An_Exploratory_Comparison_of_Australian_Colombian_German_and_Palestinian_Adolescents

\section{E 40}


Furlan, L., Sánchez, J., Heredia, D., Piemontesi, S., Illbele, A., \& Martínez, M. (2012). Estrategias de aprendizaje y afrontamiento en estudiantes con elevada ansiedad frente a los exámenes. Anuario de investigaciones de la facultad de psicología, 1, 130-141. Recuperado de http://revistas.unc.edu.ar/index.php/aifp/ article/view/2903

Gaeta, G., \& Martín, P. (2009). Estrés y Adolescencia: Estrategias de Afrontamiento y autorregulación en el contexto escolar. Studium: Revista de Humanidades, 15, 327-344. Recuperado de: http://dialnet.unirioja. es/servlet/articulo?codigo=3074506

Lazarus, R. S. y Folkman, S. (1984) Stress, Appraisal and Coping. New York: Springer Publishing Company (trad. Martinez Roca, 1986).

Lazarus, R.S.; Folkman, S. (1986). Estrés y procesos cognitivos. Martínez Roca: Barcelona, España.

Londoño, C. (2009). Optimismo y salud positiva como predictores de la adaptación a la vida universitaria. Acta colombiana de psicología, 12(1), 95-107. Recuperado De: http://www.redalyc.org/articulo. oa?id=79814903009

Londoño, N., Henao, G., Puerta, I., Posada, S., Arango, D., \& Aguirre D. (2006). Propiedades psicométricas y validación de la escala de estrategias de Coping (afrontamiento), modificada (EEC-M) en una muestra colombiana. Universitas psychologica, 5(2), 327-349. Recuperado de: http://www.redalyc.org/ pdf/647/64750210.pdf

Martín, G., Lucas, B., \& Pulido, R. (2011). Diferencias de Género en el Afrontamiento en la Adolescencia. Brocar.

Martínez, J., (2010). Estrategias de afrontamiento ante el estrés y rendimiento académico en estudiantes universitarios. Revista académica semestral, 2(18). Recuperado de http://www.eumed.net/rev/ced/18/jamg.htm

Mikkelsen, F. (2009). Satisfacción con la vida y estrategias de afrontamiento en un grupo de adolescentes universitarios de Lima. Tesis PUCP. Recuperado de http://tesis.pucp.edu.pe/repositorio/handle/123456789/416

Morales, M., \& Trianes, M. (2010). Estrategias de Afrontamiento e Inadaptación en niños y adolescentes. European journal of education and psychology. 3(2), 275-286. Recuperado de http://www.ejep.es/index. php/journal/article/viewArticle/65 
Moradi, A., Pishva, N., Ehsan, H. B., Hadadi, P., \& Pouladi, F. (2011), The relationship between coping strategies and emotional intelligence. Procedia - Social and behavioral sciences 30, 748-751. Recuperado de: http://www.sciencedirect.com/science/article/pii/S1877042811019719

Palacio, J. E., Caballero, C. C., González, O., Gravini, M., \& Contreras, K. P. (2012). Relación del burnout y las estrategias de afrontamiento con el promedio académico en estudiantes universitarios. Universitas Psychologica, 11(2), 535-544. Recuperado de: http://revistas.javeriana.edu.co/index.php/revPsycho/ article/view/583

Piemontesi, S., \& Heredia, D. (2009). Afrontamiento ante exámenes: Desarrollo de los principales modelos teóricos para su definición y medición. Anales de Psicología, 25(1), 102-111. Recuperado de: http://www.um.es/analesps/v25/ v25_1/12-25_1.pdf

Piemontesi, S., Heredia, D., Furlan, L., Sánchez, J., \& Martínez, M. (2010). Ansiedad ante los exámenes y estilos de afrontamiento ante el estrés académico en estudiantes universitarios. Anales de Psicología, 28(1), 89-96. Recuperado de http://revistas.um.es/analesps/article/view/140562/126652

Prior, M., Manzano, Vila, E., Caparrós, E., Jaume, J., \& Masergas, E. (2011). Estilos comunicativos, vinculación universitaria y adaptación psicosocial. Ediciones de la Universidad de Murcia. Recuperado en: http://www.redalyc.org/articulo. oa?id=283322847009

Rodríguez, C. (2012). Competencias académicas reporte de estrés y salud en estudiantes universitarios. Revista Electrónica de Psicología Izcala, 15(2), 553-574. Disponible en: http://www.iztacala.unam.mx/carreras/psicologia/psiclin/vol15num2/Vol15No2Art11.pdf

Saldaña, M., \& Barriga, O. (2010), Adaptación del modelo de deserción universitaria de Tinto a la Universidad Católica de la Santísima Concepción, Chile. Revista de Ciencias Sociales, 16(4), 616-628. Recuperado en: http://www.scielo.org. ve/pdf/rcs/v16n4/arto5.pdf

Salotti, P. (2006). Estudio sobre estrategias de afrontamiento y bienestar psicológico en una muestra de adolescentes de Buenos Aires. Disponible en: http://www.ub.edu.ar/investigaciones/tesinas/207_salotti.pdf

Sandín, B. (1995). El estrés. En A. Belloch Manual de psicopatología, 2. Madrid: Editorial McGraw-Hill. 
Sanjuán, P., \& Magallares, P. (2007), Estilos explicativos y estrategias de afrontamiento. Revista Clínica y Salud, 18(1), 83-98. Recuperado en: http://www.redalyc.org/articulo.oa?id=180613874006

Solís, M., \& Vidal, M. (2006). Estilos y estrategias de afrontamiento en adolescentes. Revista De Psiquiatría Y Salud Mental Hermilio Valdizan, 7(1), 33-39. Recuperado de: http://www.hhv.gob.pe/revista/2006/3\%20ESTILOS\%20Y\%20ESTRATEGIAS\%20DE\%20AFRONTAMIENTO.pdf

Tejedor, F., García, A., \& Muñoz, V. (2007). Causas del bajo rendimiento del estudiante universitario (en opinión de los profesores y alumnos). Propuestas de mejora en el marco del EEES. Revista de Educación, 342, 443-473. Disponible en http://www.ince.mec.es/revistaeducacion/re342/re342_21.pdf

Tonconi, J. (2010). en la investigación titulada factores que influyen en el rendimiento académico y la deserción de los estudiantes de la facultad de ingeniería económica de la una-puno, periodo 2009. Cuadernos de Educación y Desarrollo, 2(1). Disponible en http://www.eumed.net/rev/ced/index.htm

Tze, V., Daniels, L., Klassen, R., \& Li, J. (2013). "Canadian and chinese university students' approaches to coping with academic boredom". Learning And Individual Differences 23, 32-43. Recuperado de http://www.sciencedirect.com/ science/article/pii/S1041608012001665

Valadez, A., Bravo, M., \& Vaquero, J. (2011). Estrategias de afrontamiento empleadas por docentes universitarios. Revista Electrónica de Psicología Iztacala, 14(1), 65- 76. Recuperado de www.revistas.unam.mx/index.php/repi/article/download/24794/23323

Viñas, F., \& Caparrós, B. (2000). Afrontamiento del periodo de exámenes y sintomatología somática autoinformada en un grupo de estudiantes universitarios. Revista Electrónica de Psicología, 4(1). Recuperado de: http://www.psiquiatria.com/revistas/index.php/psicologiacom/article/view/653

Yengle, C. (2009). Adaptación a la vida universitaria de estudiantes que desertaron asociada a la relación con compañeros de estudio. UCV-Scientia, 1(1), 40-50. Recuperado de http://www.alfaguia.org/alfaguia/files/1342814505_8529.pdf 


\section{Estilos y estrategias de afrontamiento y rendimiento académico: una revisión empírica}

Resumen | En este trabajo se realiza una revisión descriptiva de investigaciones empíricas referidas a estilos y estrategias de afrontamiento y rendimiento académico en estudiantes adolescentes, tanto de forma independiente como en estudios correlacionales entre las dos variables. Se revisaron las bases de datos Dialnet, Redalyc, Scielo y Science Direct, obteniendo un total de 479 artículos, de los cuales 46 cumplían todos los criterios de inclusión (artículo de investigación, revista indexada y población adolescente). Se encontró una alta prevalencia de estudios sobre estilos y estrategias de afrontamiento y su relación con otras variables. Los estudios coinciden en correlaciones positivas entre estilos de afrontamiento activos y bienestar psicológico elevado. Coinciden también en señalar que el rendimiento académico es un fenómeno multicausado y sugieren relaciones con el afrontamiento. Sin embargo son pocas las investigaciones que correlacionan estadísticamente el afrontamiento con el rendimiento académico de manera directa.

Palabras clave | Estrategias de afrontamiento, estilos de afrontamiento, rendimiento académico y estudiantes.

\section{Citación}

Ávila-Quiñónez, A., Montaña, G., Jiménez-Arenas, D., \& Burgos, J. (2014). Estilos y estrategias de afrontamiento y rendimiento académico: una revisión empírica. Enfoques, 1(1). pp. 15 - 44

Recibido: 07/05/2014 | Aceptado: 18/06/2014

$\boldsymbol{\epsilon} \mid 44$ 